\title{
The progress of traditional Chinese medicine in the treatment of aplastic anemia
}

\author{
Ningning $\mathrm{Zhu}^{1}$, Dijiong $\mathrm{Wu}^{2}$, Baodong $\mathrm{Ye}^{2}$ \\ 'First Clinical Medical College, Zhejiang Chinese Medical University, \\ Hangzhou 310053, Zhejiang Province, China; \\ ${ }^{2}$ Department of Hematology, First Hospital Affiliated to Zhejiang Chinese Medical University, \\ Hangzhou 310006, Zhejiang Province, China
}

Address for Correspondence: Dr. Baodong Ye, Department of Hematology, First Hospital Affiliated to Zhejiang Chinese Medical University, Hangzhou 310006, Zhejiang Province, China.

E-mail: 13588453501@163.com

\begin{tabular}{|l|}
\hline Access this article online \\
\hline $\begin{array}{l}\text { Website: } \\
\text { www.intern-med.com }\end{array}$ \\
\hline $\begin{array}{l}\text { DOI: } \\
\text { 10.2478/jim-2018-0031 }\end{array}$ \\
\hline Quick Response Code: \\
\hline \\
\\
\\
\\
\end{tabular}

\section{ABSTRACT}

Aplastic anemia (AA) is a common hematologic disease that is characterized by hematopoietic failure of the bone marrow and pancytopenia of the peripheral blood, which can be divided into severe and non-severe aplastic ane-mia, or acute and chronic aplastic anemia according to the severity of the disease and the progress of the clinical course. During the past years, the advantages of Traditional Chinese Medicine (TCM) on the treatment of AA have been well clarified and its theory system has been improved as well. This review is mainly on representing the pathogenesis, therapeutic principle and method, research progression, and advantages of TCM on AA.

Key words: aplastic anemia, traditional Chinese medicine treatment, Chinese drugs, review

\section{INTRODUCTION}

Aplastic anemia (AA) is a bone marrow hematopoietic dysfunction disease caused by one or more factors, which is characterized by low bone marrow nucleated cell proliferation and peripheral blood pancytopenia, without abnormal cell infiltration or myelofibrosis. ${ }^{[1]}$ The main clinical manifestations consist of different degrees of anemia, hemorrhage and infection. ${ }^{[2]}$

Up to now, the Camitta standard is still used internationally. The severity of AA is defined according the blood count parameters and bone marrow findings, which can be divided into very severe aplastic anemia (VSAA), severe aplastic anemia (SAA) and non-severe aplastic anemia (NSAA). ${ }^{[3]}$ According to the progress of the disease, aplastic anemia is further divided into acute aplastic anemia and chronic aplastic anemia in China. Camitta classification emphasizes the severity of hematopoietic failure, while the domestic standard also emphasizes the progress of such failure. ${ }^{[4]}$

\section{APLASTIC ANEMIA IN TCM THEORY}

The origin of aplastic anemia in TCM There is no Traditional Chinese Medicine (TCM) disease name for aplastic anemia in the classic Chinese medicine. However, according to the manifestation of aplastic anemia, acute aplastic anemia is mostly attributed to "acute consumptive disease" and "bone marrow deficiency". Chronic aplastic anemia belongs to "blood disease", "chronic consumptive disease" and "blood deficiency". ${ }^{[5]}$ The name "bone marrow deficiency" first appeared in the Huangdi's Canon of Medicine, and was described as "the yin and yang are exhausted, the blood and qi are in deficiency". The clinical manifestation includes anemia and hemorrhage, which are similar to aplastic anemia. People with insufficient of qi and blood always come along with marrow breakdowns. ${ }^{[6]}$ In 1950s, the name and diagnostic criteria for "aplastic anemia" was also applied in Chinese medicine. In 2007, the national AA collaboration group 
meeting in Wuhan unified AA into the category of "bone marrow deficiency" in traditional Chinese medicine. "Bone marrow" stands for the position of the disease, and "deficiency" stands for the condition and nature of disease. ${ }^{[7]}$

\section{Etiology and pathogenesis of aplastic anemia}

At present, the pathogenesis of aplastic anemia is still not fully elucidated, the consensus is that AA is related to the abnormal immunity, defected hematopoietic microenvironment, shortage or dysfunction of hematopoietic stem cell, as well as genetic abnormality. Large numbers of studies have proved that $\mathrm{T}$ lymphocytes have a close relationship with hematopoietic failure. ${ }^{[8]}$ Based on this, the extensive application and good efficacy of immunosuppressive also confirmed the significance of immunopathology in the development of aplastic anemia. ${ }^{[9-10]}$

The importance of bone marrow to functional qi and blood had been recognized two thousand years ago in TCM. Huangdi's Canon of Medicine showed "Bone marrow firmed, then qi and blood are abundant", which indicated that the location of disease is marrow. The TCM etiological factors consist of hereditary factors, exogenous pathogens, improper diet, seven emotions imbalance and the poison of medicine, which impair the internal organs, cause the consumption of essence and marrow, and lead to the deficiency of qi and blood. ${ }^{[1]}$

In 1950s, patients were treated based on the categorization of patterns/syndromes according to the state of qi and blood. In 1960s, TCM physicians realized the importance of syndrome differentiation of zang-fu viscera. ${ }^{[12]}$ Before 1989, the treatment and clinical researches were carried out based on the theory of "spleen deficiency", which focus on the "spleen"; however, the efficacy was limited. Since then, the TCM hematologists started exploring and practicing with the guidance of "kidney deficiency" as the main characteristic of the disease, and the theoretical system and treatment strategy of aplastic anemia were established, ${ }^{[13]}$ which exerted a much better clinical efficacy. Based on the principal of "kidney deficiency", there are also some additional perspectives of the pathogenesis in TCM, which maybe suitable for different situations or stages of the disease. The most popular perspectives can be summarized as follows:

\section{The deficiency of kidney essence}

According to the theory of TCM, the main functions of kidney are store essence, control the development and reproduction, dominate the bone and manufacture the marrow. The essence and blood comes from the same source, and the essence can transform into blood.
Kidney essence is the source of bone marrow. Based on the traditional Chinese medicine theory and the progress of modern medical research, Ma Ming established a new theory of "ministerial fire", and clarified that kidney deficiency is crucial to the development of chronic aplastic anemia. $^{[14]}$

Ministerial fire is the original motivity of kidney in TCM, and the functional hematopoiesis is the manifestation of the same. During the physiological stage, the kidney is strong and with exuberant essence, which can be transformed into blood. Zhou Yuhong conceived that although the clinical syndromes of $\mathrm{AA}$ are characterized as deficiency of qi and blood, the primary cause of AA is the deficiency of vital energy in kidney, which impair the generation of $\mathrm{Y}$ in essence and blood. ${ }^{[15-16]}$

\section{Spleen-stomach weakness}

In the theory of TCM, the spleen is the basis of postnatal life, which governs the transportation and transformation of nutrient substance and is in charge of the replenishment of kidney essence. Shen Yiping believed that the deficiency of spleen and kidney was the basis of the pathogenesis of AA in TCM. When kidney Yang is in deficit, the spleen and stomach are incapacitated, and the cereal essence cannot be transformed into essence and blood. ${ }^{[17-19]}$

\section{The deficiency of liver blood}

The blood of liver is derived from the postnatal essence, which is nourished by the nutrient substance of spleen. And the function of spleen and stomach depends on liver's governing free flow of qi. Only with a functional liver, the qi movement is smooth and hematopoiesis works. The consumption of kidney essence lead to the deficiency of blood and the dysfunction of liver, which can potentially cause the loss of kidney essence and lead to consumptive disease. ${ }^{[20]}$

\section{Generation of static blood}

When qi and blood are both deficient, the pulse is in poor nutrition, and the circulation of blood is not smooth. In that case, blood stasis appears over time. In the other way, when yin blood is depleted, heat is endogenous, the movement of blood is overflowing and stagnant, and marrow is impaired as well. ${ }^{[21]}$ Qiu et al. ${ }^{[22]}$ conceived that some of the symptoms of aplastic anemia, such as dark complexion and skin malformation, are dialectically attributed to the presence of blood stasis in TCM.

At present, there are two main aspects about the mechanism of blood stasis in the formation of aplastic anemia. Firstly, it is caused by deficiency. Yang et al. ${ }^{[23]}$ believe that the formation of blood stasis is due to either the lack of kidney yin; in that case, the pulse is empty and even exhausted. Or 
it is due to deficiency of kidney yang and cold is coagulated in pulse; hence, blood flow is stagnated. The second cause is pathogenic qi. The pathogenic qi is invaginated to steam and suffocate blood or disturbing the flow of blood. ${ }^{[2]}$ Static blood could appear at any pathogenesis process of aplastic. Deng et al. ${ }^{[25]}$ had approved that all the AA patients with different pathogenesis may have abnormal nail microcirculation. As a pathological product of $\mathrm{AA}$, blood stasis can occur in any stage of the disease, which can become a pathogenic factor to aggravate bleeding. Therefore, when the disease condition is not stable, static blood would be difficult to cure. ${ }^{[26]}$

\section{Heat toxin}

According to the TCM theory, when heat toxin enter into the nutrient-blood, it will lead to the consumption of blood and marrow. Meanwhile, heat also disturbs the flow of blood and causes various kinds of hemorrhage. According to the classical medical records and years of clinical practice research, Liu et al. ${ }^{[27]}$ conceived that bone marrow is enmeshed by heat toxin and summarized the theory that "treatment of aplastic anemia from the clearance heat toxin".

\section{The Syndrome Differentiation of AA in TCM}

Currently, the classification of aplastic anemia is mainly in accordance with the standards formulated by the professional hematology committee of traditional Chinese and western medicine in 1989. The conference unified aplastic anemia into four types: "Heat into nutrient blood", "Kidney Yin deficiency", "Kidney Yang deficiency" and "Deficiency of both Kidney Yin and Yang". Acute aplastic anemia is mainly characterized by "Heat into nutrientblood" type, and "Kidney Yin deficiency", "Kidney Yang deficiency" and "Deficiency of both Yin and Yang" mostly belong to chronic aplastic anemia. ${ }^{[28]}$ This classification has been recognized and widely used in China. In 2008, the "Guidelines for the diagnosis and treatment of common diseases in Chinese medicine" ${ }^{[29]}$ were issued by the association of Chinese Medicine and this increased the four types of AA in TCM, consisting of "Yang deficiency of both spleen and kidney", "Yin deficiency of both liver and kidney", "Heat toxin" and "Blood heat" type. The guidelines enriched the aplastic anemia syndrome's evolution characteristics, but the limitations for clinical practice still exist. In 2010, the diagnosis and treatment plan for 95 diseases in 22 specialties in $\mathrm{TCM}^{[30]}$ was promulgated by the State Administration of TCM, of which, the aplastic disability was divided into "kidney Yin deficiency", "kidney Yang deficiency", "deficiency of both Yin and Yang", "Excessive heat toxin" and "fire excess from Yin deficiency", the five types were basically recognized and promoted by hematologists. ${ }^{[31]}$

\section{TREATMENT OF AA IN TCM}

The principle of "symptomatic treatment in acute condition, radical treatment in chronic case" is followed on the treatment of AA in TCM. At present, the therapeutic methods are based on "tonifying kidney" and regulating organs, supplemented by the methods of clearing away heat and detoxifying, cooling blood to stop bleeding, and removing blood stasis. ${ }^{[32]}$

\section{Syndrome differentiation and treatment of CAA Tonifying kidney}

Mainly, the pathogenesis of CAA is kidney deficiency. At present, TCM hematologists have reached a domestic consensus on kidney-tonifying as the core process during the treatment of CAA in TCM. ${ }^{[33]}$ Warming kidney Yang is used to treat patients with kidney Yang deficiency syndromes, and nourishing kidney Yin is used to treat patients with kidney Yin deficiency syndromes. Su et al. ${ }^{[34]}$ found that after a 6 month-treatment based on kidney tonifying, the peripheral blood get improved, and the leukocyte and platelet count increased at different degrees. Meanwhile, he also reported that the effect of kidney Yang deficiency group was obviously better than the kidney Yin deficiency group. According to the multicenter randomized controlled trials, Shen et al. ${ }^{[35]}$ found that Chinese medicines can adjust the balance of Th cells, regulate the expression of IFN- $\gamma$, IL-2, IL-4, IL- 6 , promote the recovery of hemogram index and improve the bone marrow microenvironment. ${ }^{[3]}$

\section{Tonifying spleen}

CAA patients always suffer from low appetite, lassitude, asthenia, and hemorrhage. All these symptoms are related to deficiency of spleen in TCM. The spleen is located in the middle energizer, the main functions of spleen are control blood and transformation. It is widely applied to treat the AA by tonifying kidney and spleen at the same time. ${ }^{[37]}$ Zeng Qing adopted a self-designed prescription, which mainly contains dogwood, epimedium herb, psoralea corylifolia linn, ginseng, astragalus for the treatment of CAA with deficiency of spleen and kidney Yang. It was reported that the formula can significantly improve the anemia symptoms of patients, enhance hemogram index, obviously reduce $\mathrm{CD}^{+}$and increase $\mathrm{CD}^{+}$levels, as well as promote the recovery of $\mathrm{CD}^{+}$level and $\mathrm{CD} 4^{+} / \mathrm{CD}^{+}$ratio. ${ }^{[38,39]}$

\section{Regulating liver}

Based on the pathogenesis characteristics of CAA, Wang Haixia ${ }^{[40]}$ applied "kidney-tonifying and regulating liver therapy" to treat chronic aplastic anemia patients. "Xiaoyao powder" achieved a good clinical effect with the combination of tonify method and harmonization method. 


\section{Reducing phlegm and dissipating blood stasis}

Zhou Yuhong summarized the pathogenesis of CAA as follows: kidney-deficiency, pathogenic factors, phlegm and blood stasis. Kidney essence deficiency and viscera damage lead to dysfunction of transportation and transformation; the bone marrow is dried up, the blood is exhausted, and both qi and blood are deficient. ${ }^{[41]}$ Laboratory studies have shown that blood-activating and stasis-eliminating compound can improve the microenvironment of bone marrow, accelerate its metabolism, adjust the vital qi in the body and improve the cellular immune function, which can benefit the growth of hematopoietic stem cells and increase the capacity of hematopoietic tissue. ${ }^{[42]}$

\section{Detoxication}

The treatment of aplastic anemia should firstly focus on the primary cause, to eliminate the pathogenic qi and tonify marrow. According to the stage of the pathogenic qi, patients are treated with detoxification, with the combination of heat clearing, removing blood stasis, benefiting qi and gill toxicity, nourishing Yin and warming Yang. ${ }^{[43]}$ Zhao et al. ${ }^{[4]]}$ treated 34 cases of aplastic anemia with kidney-tonifying and blood detoxification, the total effective rate was $82.35 \%$, which was higher than pure kidney-tonifying group.

\section{Different-stage treatment of SAA in TCM}

Acute aplastic anemia is characterized with short clinical course, acute disease, and clinical apostasis, and the immunosuppression as well as hematopoietic stem cells are the main treatment. According to the observation, we found that anti-lymphocyte immunoglobulin is characterized by heat property, which can consume qi and Yin. If ATG is effective and hemogram index gradually recovers, the syndrome of Yin deficiency should be corrected first. For patients receiving ATG treatment, "cool - warm - hot" is currently used in clinical treatment in different stages. At the primary stage, there are symptoms of excessive heat a or Yin deficiency, and "cool" treatment is given for detoxification and nourishing Yin (this stage is usually before ATG treatment). If the infection is controlled, and the heat is cleared, symptoms of both deficiency of qi and Yin are shown, then the product of strengthening spleen and nourishing kidney can be added for nourishing Yin and nourishing essence (this stage is usually within 3 months of ATG treatment). When the condition is stable, Yin gradually recovers and the symptoms of Yang deficiency appear, warm kidney and impotence products are added to promote bone marrow hematopoiesis (this stage is usually 3 months after the ATG treatment). ${ }^{[45]}$

\section{MODERN RESEARCH: THE MECHANISM OF TCM IN TREATING}

\section{APLASTIC ANEMIA}

\section{Regulation of cellular immunity}

$\mathrm{Ni}$ et al. ${ }^{[46]}$ found that both "Xue Fu Sheng" and cyclosporin can down-regulate the expression of CD28 in T cells, and the combined use of both is better than the single treatment, indicating that the "Xue Fu Sheng" can regulate the cellular immune function of the aplastic mice.

\section{Regulation of cytokines}

Ding et al. ${ }^{[47]}$ found that Kidney-Nourishing decoction can promote the total production of IL-3 factor, which promoted the normal proliferation and differentiation of hematopoietic stem cells. Zheng et al..$^{[8]}$ found that Stasiseliminating and Kidney-tonifying decoction can promote the repair of bone marrow microenvironment in AA mice, reduce the release of negative regulation factors of hematopoiesis, and promote the recovery of hematopoietic function.

\section{Improvement of hematopoietic microcirculation}

After the clinical randomized trials, Sun Zhongliang ${ }^{[49]}$ found that the proportion of peripheral blood reticulocyte, neutrophil, platelet, and hemoglobin bone marrow hematopoietic cells in the ligustrazine group was significantly increased compared with that in the control treatment group. Furthermore, the number of bone marrow microvessels, the volume of hematopoietic tissue and the collection of fibroblasts were significantly higher than those in the pretreatment and basic treatment groups. The results demonstrated that ligustrazine could improve the bone marrow microenvironment in AA patients.

\section{THE ADVANTAGES OF TCM}

\section{Reduce Complications}

The study reported that the occurrence rate of infection in patients with aplastic anemia during hospitalization was up to $46.4 \%$, and upper respiratory tract infection was common. ${ }^{[50]}$ The invasion of bacteria and virus could trigger the expression of inflammatory factors, and the hematopoietic function of bone marrow was shocked again. The patients with aplastic anemia had the lowest platelet recovery and long-term risk of hemorrhage. Following the therapeutic principle of syndrome and disease differentiation, treatment that is based on tonifying spleen and kidney can enhance patients' ability of the invaded, reduce the incidence of colds, and effectively reduce the various hemorrhage symptoms. ${ }^{[1]}$

\section{Immunoregulation}

The research showed that "Xue Fu Sheng" (main ingredients: buckhorn gum, astragalus, angelica sinensis, 
ligustrum lucidum, etc.) can effectively improve the disorder of $\mathrm{T}$ cell subgroup, correct the $\mathrm{CD}^{+} / \mathrm{CD}^{+}$ inversion phenomenon, and increase the content of $\mathrm{CD}^{+}$ cells in bone marrow. ${ }^{[52]}$ "Kidney-tonifying therapy" can significantly inhibit the mRNA expression of TNF - $\alpha$ and IFN $-\gamma$ in the bone marrow, and up-regulate the levels of IL-3 and stem cell factor (SCF), ${ }^{[53]}$ which is conducive to the recovery of hematopoietic function.

\section{Reduce adverse reactions of western drugs and improve quality of life}

Early intervention of treatment in TCM can regulate the overall balance of Yin and Yang, increase efficiency and reduce toxicity. $\mathrm{Xu}$ et al. ${ }^{[54]}$ observed 328 patients with chronic aplastic anemia, iKanglilong control group (163 patients) showed 27 cases of liver damage, 51 cases of varying degrees of acne, hairyness, and hoarseness. At the same time, in the group combined with TCM, none of the above adverse reactions occurred, and the effect was significantly increased $\left(87.3 \%\right.$ vs. 62.6\%). Yuan et al. ${ }^{[5]}$ found that by supplementing liver and kidney, nourishing qi blood, patients can significantly get their symptoms such as complexion, dizziness, and shortness of breath relieved; hence, their quality of life was improved.

\section{SUMMARY}

Aplastic anemia is a common hematological disease, with a long disease course and treatment. During the past years, a large number of experimental studies have provided objective basis for the treatment of aplastic anemia with TCM. However, there are still some deficiencies and problems, such as the discussion and induction of TCM etiology and pathogenesis is not comprehensive, the research methods are not objective enough. A large amount of clinical research data is still needed for the therapeutic mechanism and the theory of synergistic and attenuating toxicity. The prospective, randomized, and multicenter studies are the main research directions in the future. Innovation is needed in the scientific research in TCM. The TCM research that meets the clinical needs, aims to improve the level of prevention and treatment of major diseases in China, will get further developed.

\section{Source of Foundation}

The present study was supported by the Special Research Funds for Traditional Chinese Medicine Industry from State Administration of Traditional Chinese Medicine (201407001) and National Natural Science Foundation Project (grant No. 81774092).

\section{Conflict of Interest}

The authors have no conflicts of interest to declare.

\section{REFERENCES}

1. Killick SB, Bown N, Cavenagh J, Dokal I, Foukaneli T, Hill A, et al. Guidelines for the diagnosis and management of adult aplastic anaemia. Br J Haematol 2016; 172: 187-207.

2. Brodsky RA, Jones RJ. Aplastic anaemia. Lancet 2005; 365: 1647-56.

3. Fu R. Chinese expert consensus on the diagnosis and treatment of aplastic anemia. Chin J Hematol 2017; 38: 1-5.

4. Lin SY. Treatment of aplastic anemia by integrated Chinese and western medicine. Chin J Pract Internal Med 2016; 36: 358-60.

5. Qi CJ, Cai LM, Xu MD, Kuang ZZ, Zhang N. The Research Progress on Preventive Treatment for Aplastic Anemia by Traditional Chinese Medicine. Prog Mod Biomed 2015; 15: 5174-7.

6. Zuo XY, Wang AD, Liu BS. Pathogenesis analysis of aplastic anemia. J Tianjin Univ Tradit Chin Med 2017; 36: 248-50.

7. Chen XY, Ma R, Li DY. Recommendations for common blood diseases in Chinese medicine. Chin J Integr Tradit West Med 2009; 29: 1040-1.

8. Xiao Y, Zhao S, Li B. Aplastic anemia is related to alterations in $\mathrm{T}$ cell receptor signaling. Stem Cell Invest 2017; 4: 85.

9. Zeng Y, Katsanis E. The complex pathophysiology of acquired aplastic anaemia. Clin Exp Immunol 2015; 180: 361-70.

10. Liu CY, Fu R, Wang HQ, Li LJ, Liu H, Guan J, et al. Fas/FasL in the immune pathogenesis of severe aplastic anemia. Genet Mol Res 2014; 13 : 4083-8.

11. Sun Y, Ye BD, Zhou YH. Discussions on etiology and pathogenesis of aplastic anemia from the theory of TCM. J Tradit Chin Med 2015; 56: 1073-5.

12. Wei XL, Dai XB, Zhou YM. Retrospect and Reflection on syndrome differentiation and treatment of Aplastic Anemia in TCM . Shanghai J Tradit Chin Med 2012; 46: 4-6.

13. Wu DJ, Shen YP, Hu ZP, Ye BD, Lin SY, Wu LQ, et al. Reflection on syndrome differentiation and treatment of Severe Aplastic Anemia in TCM. J Zhejiang Chin Med Univ 2017; 41: 262-4.

14. Ma M. Preliminary study on the pathogenesis and treatment of hematopoietic diseases. J New Chin Med 1995; 27: 7-8.

15. Sun CL, Zhou YH. Research on the mechanism and treatment strategies of integration of traditional Chinese and western medicine for aplastic anemia. Anhui Med Pharm J 2008; 12: 887-9.

16. Zhou JY, Liu BW. Research progress in characteristics of pathogenesis and treatment in traditional Chinese medicine of aplastic anemia. Yunnan J Tradit Chin Med Materia Med 2016; 37: 74-5.

17. Pan QW, Shen YP. Shen yiping's experience in treating chronic aplastic anemia. Zhejiang J Integr Tradit Chin West Med 2017; 27: 1-3.

18. Zhang YF. Treatment of chronic aplastic anemia by Chinese medicine. Asia Pac Tradit Med 2017; 13: 67-8.

19. Li DJ, Ke WJ. Ke Weijun's experience in treating chronic aplastic anemia. J Emerg Tradit Chin Med 2014; 23: 1289.

20. Wu XY. Theoretical Discussion on the Treatment of Chronic Aplastic Anemia from Liver, Spleen and Kidney. J Guiyang Tradit Chin Med Coll 2009; 31: 1-2.

21. Chen L, Yang WH, Yang WY, Chen YQ. Treatment of acute aplastic anemia from kidney deficiency, phlegm and blood stasis. J Tradit Chin Med 2014; 55: 1427-9.

22. Li YH, Lan H, Hu XY, Gu XK, Qiu HM. Qiu Heming's experience in treating chronic aplastic anemia. J Guangzhou Univ Tradit Chin Med 2015; 32: 940-2.

23. Yang WH, Wan ZZ, Dai XM, Yang XS. Study on the Mechanism of Bushen Huoxue Recipe in Treating Aplastic Anemia. Tianjin J Tradit Chin Med 1993; 20: 22-3.

24. Yang GB, Liu YF. Discussion on the etiology and pathogenesis of chronic aplastic anemia. Guangxi J Tradit Chin Med 2016; 39: 48-9.

25. Deng YA, Yan WR, Gao D, et al. Study on blood stasis syndrome of aplastic anemia. Chin J Integr Tradit West Med Intensive Crit Care 1997; $4: 62-5$. 
26. Wu DJ, Shen YP, Zhou YH, Hu ZP, Wu LQ, Zhang Y. Discussion on the View of "Tan Yu Tong zhi" in Acute Aplastic Anemia. J Tradit Chin Med 2010; 51: 91-2.

27. Xu YW, Teng YY, Liu QF, Liu DT. Professor LIU Datong's treatment of aplastic anemia from toxin. J Changchun Univ Chin Med 2016; 32: 481-2.

28. Wu ZX. Minutes of the 3rd National Conference on Integrated Chinese and Western Medicine on Hematology. Chin J Integr Tradit West Med 1992; 12: 319-20.

29. China Association of Chinese Medicine. Guidelines for the diagnosis and treatment of common diseases in Chinese medicine. Beijing: China Press of Traditional Chinese Medicine, 2008: 209-11.

30. State Administration of Traditional Chinese Medicine of the People's Republic of China. The diagnosis and treatment plan for 95 diseases in 22 specialties in TCM. Beijing: China Press of Traditional Chinese Medicine, 2011: 328-30.

31. Wu DJ, Ye BD, Ji CH, Chen D, Zhang Y, Sheng YP, et al. Research status of clinical practice guidelines for aplastic anemia and amendment and thinking of Traditional Chinese Medicine guidelines. Zhong Yi Za Zhi 2014; 55: 274-8.

32. Tao JH. Data Mining Research on TCM Syndrome Classification of Aplastic Anemia. Shenyang: Liaoning University of Traditional Chinese Medicine, 2011: 1-51.

33. Tang XD, Zhang SS, Ma R, Xu YG, Yang XH, Xiao HY, et al. Study on Immunological Mechanism and Curative Effect Prediction Index of Aplastic Anemia Treated by Combination of Traditional Chinese Medicine and Western Medicine. China J Tradit Chin Med Pharm 2012; 27: 1101-5.

34. Su XL, Xia YY, Chen SN. Clinical observation on kidney-treating therapy for chronic aplastic anemia. Pract Pharm Clin Remedies 2017; 20: 287-9.

35. Wu D, Shen Y, Ye B, Fang B, Lin S, Chen Z, et al. Efficacy and advantages of modified Traditional Chinese Medicine treatments based on "kidney reinforcing" for chronic aplastic anemia: a randomized controlled clinical trial. J Tradit Chin Med 2016; 36: 434-43.

36. Ye B, Chen D, Wu D, Wu X, Zhang X, Zhou Y, et al. Effect of kidneyreinforcing, blood-activating and stasis-removing recipes on adhesion molecule expression of bone marrow mesenchymal stem cells from chronic aplastic anemia patients. J Tradit Chin Med 2012; 32: 596-603.

37. Wu DJ, Zhou YH, Shen YP. TCM Advantages of Chronic Aplastic Anencia. Chin Arch Tradit Chin Med 2012; 30: 500-2.

38. Zeng Q, Cheng WM. 30 Cases of Chronic Aplastic Anemia with Asdthenic Splenonephro-yang Treated by Shenyubuxuefang. J Guangxi Tradit Chin Med Univ 2005; 8: 18-9.

39. Zeng Q, Cheng WM, He A. Research of Shenyu Buxue Formula on Clinical Effect and Immunological Mechanism for Patients suffering from Chronic Aplastic Anemia of Spleen and Kidney Yang Deficiency. Guangxi J Tradit Chin Med 2018; 41: 3-5, 40.

40. Gu SW, Cui C, Wang JH, Yang GM, Wang SQ, Wang HX. Effects of kidney-tonifying and hepatosplenic therapy on telomere activity of peripheral blood mononuclear cells in patients with chronic aplastic anemia. Tianjin J Tradit Chin Med 2017; 34: 809-12.
41. Zheng RZ, Wu DJ, Ye BD, Shen YP, Zhou YH. ZHOU Yuhong's Experience in Treating Chronic Aplastic Anemia in Combination of Syndrome Differentiation and Constitution Differentiation. J Tradit Chin Med 2017; 58: 1997-2000.

42. Yu G, Shi YQ . Study on the application of promoting blood circulation and removing blood stasis in aplastic anemia. J Zhejiang Chin Med Univ 199; 23: 57-8.

43. Xu YW, Liu QF, Teng YY, Liu DT, Gao HB. Standardized treatment program for the application of traditional Chinese medicine in the treatment of aplastic anemia with Professor LIU Datong. J Changchun Univ Chin Med 2017; 33: 277-8.

44. Zhao XG, Liu DZ, Sun YY, Sun WZ. Clinical Research on Treating Aplastic Anemia by Tonifying the Kidney, Promoting Blood Circulation and Detoxicating. J Gansu Chin Med Univ 2001; 18: 22-4.

45. Wu DJ, Hu ZP, Shen YP, Zhou YH, Shen JP, Wu LQ, et al. Approach of staging evidences of acute aplastic anemia. China J Tradit Chin Med and Pharm 2010; 45: 1099-101.

46. Ni HW. Effection of Xuefusheng on the expression of CD28 and CTLA4 and the levels of IFN- $\gamma$ and TNF- $\alpha$ in bone marrow of mice with immune-mediated aplastic anemia.Nanjing: Nanjing University of Chinese Medicine, 2009: 1-43.

47. Ding JY, HUang ZC, Zhou YM, Huang T, Xue ZZ, Sun GL, et al. Effects of "Kidney-Nourishing Decoction" on IL-3 and IFN- $\gamma$ in Immune-Mediated Aplastic Anemia Mice. Acta Univ Tradit Med Sinensis Pharmacologiaeque Shanghai 2003; 9: 52-4.

48. Zheng J, Liu Q, Wang ZR, Li JC, Wang W, Ma AL, et al. Effect of Stasiseliminating and Kidney-tonifying Decoction on CFU-F,IL-2 and IFN $\gamma$ in Aplastic Anemia Mice. J Anhui Univ Chin Med 2004; 23: 33-5.

49. Sun ZL.The effects of chuanxiongzine on bone marrow microenvironment in aplastic anemiaJ.. Cent China Med J 2009; 33: 194-6.

50. Tao LJ, Chen SQ, Wang XC. Nosocomial Infection for Aplastic Anemia and Its Risk Factors. Chin J Nosocomiol 2010; 20: 342-4 .

51. Xiao Y, Feng J, Yin Y, Sun WZ. Clinical Study on Treatment of Chronic Aplastic Anemia with Buxue Shengxue Granule. Inf Tradit Chin Med 2008; 25: 54-6.

52. Ji JM, Zhang YC, Sheng Q, Liu J, Zhu GR, Zhu QL, et al. Investigation on the mechanism of chronic aplastic anemia treated with herbs complex Xue-Fu-Sheng. Chin J Biochem Pharm 2010; 31: 205-7.

53. Xu RR, Wang Y, An YJ, Yu ZF, Dai XM. Effect of Chronic Aplastic Anemia Treated with Promoting Kidney and Tonifing Bone Method on the Expression of Hematopoiesis Regulation Factors IL-3, SCF, IFN- $\gamma$ and TNF- a mRNA. Chin J Inf Tradit Chin Med 2007; 14: 12-4.

54. Xu WJ, Yang SL, Li Q, Di HX, Qiao ZJ. Clinical observation of treatment of aplastic anemia with Zaizhang Shengxue Granule. Chin J Exp Tradit Med Formul 2010; 16: 131-2.

55. Yuan JQ, Yuan LN, Zhou KJ. Clinical Study on Treatment of 120 Cases of Aplastic Anemia with Shengxue Pills. China Mod Med 2009; 16: 59-60.

How to cite this article: $\mathrm{Zhu} N, \mathrm{Wu} D$, Ye B. The progress of traditional Chinese medicine in the treatment of aplastic anemia. J Transl Intern Med 2018; 6: 159-64. 